\title{
RECURSIVE PROPERTIES OF TRANSFORMATION GROUPS
}

W. H. GOTTSCHALK AND GUSTAV A. HEDLUND

Introduction. If a continuous flow, the transformations of which are denoted by $f^{t}$, is defined in a topological space $X$, a point $x$ of the space can be classified according to the behavior of the continuous orbit of the point. Among the types of points which have been considered are the periodic points, almost periodic points and recurrent points. If we fix the value of the parameter $t$, we obtain a transformation of the space $X$ into itself. This transformation and its iterates determine a "discrete" flow and the "discrete" orbit of a point. Again a point of the space can be classified according to the behavior of the discrete orbit of the point. The question then arises as to whether a point belongs to the same class in the two cases.

Since the continuous orbit of a point contains the discrete orbit, many properties are retained when we pass from the discrete flow to the continuous flow. For example, if a point is periodic with respect to the discrete flow, it is clearly periodic with respect to the continuous flow. It is in the passage from the continuous to the discrete flow that the results are more difficult to predict.

In this note, the problem is generalized by replacing the parameter space of reals by an additive, abelian, locally compact, topological group. The action of such a transformation group $T$ on a point and the action of certain subgroups $G$ on the point are then related. It is shown that a general recursive property of $T$ carries over to $G$ (Theorem 1). It follows immediately that in the case of a continuous flow, if a point is either almost periodic, recurrent, or strongly recurrent with respect to the continuous flow, it retains this property with respect to the discrete flow determined by fixing the parameter $t$. It also follows that these properties carry over from a discrete flow to a sub-discrete flow. (For recurrence and almost periodicity, this was first proved by Gottschalk [2 $]^{1}$ and subsequently extended to discrete semi-flows by Erdös and Stone [1].)

Theorem on recursive points. Let $X$ be a topological space and let $T$ be an additive abelian locally compact topological group. Let $f$ be a continuous transformation of $X \times T$ into $X$. We agree to write $f^{t}(x)$ in place of $f(x, t) \quad(x \in X, t \in T)$ whenever we wish. Furthermore, let $f$

Presented to the Society, August 23, 1946; received by the editors April 9, 1946.

1 Numbers in brackets refer to the Bibliography at the end of the paper. 
define a transformation group; that is to say, suppose $f^{0}(x)=x$ and $f^{s} f^{t}(x)=f^{t+s}(x)(x \in X ; t, s \in T)$.

Let there be distinguished in $T$ certain nonvacuous sets, called $a d$ missible, which satisfy this condition: If $A$ is an admissible set and if $B$ is a set in $T$ such that for some compact set $C$ in $T$ we have $a \in A$ implies $B \cap(a+C) \neq \varnothing$, then $B$ is an admissible set. If $x \in X$ and if $S$ is a set in $T$, then $x$ is said to be recursive with respect to $S$ provided that if $U$ is a neighborhood of $x$, then there exists an admissible set $A$ such that $A \subset S$ and $f(x, A) \subset U$.

We suppose throughout that $x$ is a fixed point of $X$ and that $G$ is a fixed topologically closed subgroup of $T$ such that $T=F+G$ for some compact set $F$ in $T$. We define $H$ to be the set of all elements $t$ of $T$ such that $x \in \bar{f}(x, t+G)$, where $\bar{f}(x, t+G)$ denotes the closure of $f(x, t+G)$.

LEMMA 1. $H$ is a topologically closed semi-group $(H+H \subset H)$ which contains $G$.

Proof. Suppose $t, s \in H$ and $U$ is a neighborhood of $x$. There exists an element $p$ in $G$ such that $f(x, t+p) \in U$. Select a neighborhood $V$ of $x$ for which $f(V, t+p) \subset U$. For some element $q$ in $G, f(x, s+q) \in V$. Hence, $f(x, t+s+p+q) \in U$. Thus $H$ is a semi-group. Obviously, $G \subset H$.

Suppose $t \in \bar{H}$ and $U$ is a neighborhood of $x$. Choose neighborhoods $V$ of $x$ and $W$ of 0 for which $W=-W$ and $f(V, W) \subset U$. Let $s \in(t+W) \cap H$. For some element $p$ in $G, f(x, s+p) \in V$. Now $f(x, t+p)=f^{t-s} f(x, s+p) \in f^{t-s}(V) \subset U$. Thus $H$ is topologically closed and the proof is completed.

Lemma 2. There exists a compact set $E$ in $H$ such that $H=E+G$.

Proof. There exists a compact set $F$ in $T$ such that $T=F+G$. Define $E=F \cap H$. Using Lemma 1, we see that the set $E$ has the required properties.

LEMmA 3. If $H$ is a group and if $U$ is a neighborhood of $x$, then there exists a compact set $C$ in $H$ such that $f(x, C) \subset U$ and $t \in H$ implies $(t+C) \cap G \neq \varnothing$.

Proof. By Lemma 2, there exists a compact set $E$ in $H$ such that $H=E+G$. Define $D=-E$. Now $t \in H$ implies $(t+D) \cap G \neq \varnothing$. For each element $d$ in $D$, there exists an element $t_{d}$ in $G$ so that $f\left(x, d+t_{d}\right)$ $\in U$ and, hence, there exists a compact closed neighborhood $W_{d}$ of $d$ for which $f\left(x, W_{d}+t_{d}\right) \subset U$. Cover $D$ by finitely many neighborhoods 
$W_{d_{1}}, \cdots, W_{d_{n}}$. Define $C=H \cap \bigcup_{i=1}^{n}\left(W_{d_{i}}+t_{d_{i}}\right)$. The set $C$ has the required properties.

LEMMA 4. If $H$ is a group and if $x$ is recursive with respect to $H$, then $x$ is recursive with respect to $G$.

Proof. Let $U$ be a neighborhood of $x$. By Lemma 3, there exists a compact set $C$ in $H$ such that $f(x, C) \subset U$ and $t \in H$ implies $(t+C) \cap G \neq \varnothing$. Choose a neighborhood $V$ of $x$ for which $f(V, C) \subset U$. There exists an admissible set $A$ such that $A \subset H$ and $f(x, A) \subset V$. Hence, $f(x, A+C) \subset U$. Define $B=(A+C) \cap G$. The set $B$ is admissible and contained in $G$. Also $f(x, B) \subset U$. The proof is completed.

Lemma 5. If $M, N$ are subsets of $T$ such that $M$ is compact and $x \in \bar{f}(x, M+N)$, then there exists an element $m$ in $M$ for which $x$ $\in \bar{f}(x, m+N)$.

PRoof. Suppose the contrary. Then for each element $m$ in $M$ we can find a neighborhood $U_{m}$ of $x$ such that $f(x, m+N) \cap U_{m}=\varnothing$; selecting neighborhoods $V_{m}$ of $x$ and $W_{m}$ of 0 so that $W_{m}=-W_{m}$ and $f\left(V_{m}, W_{m}\right) \subset U_{m}$, we have $f\left(x, m+W_{m}+N\right) \cap V_{m}=\varnothing$. Choose finitely many neighborhoods $m+W_{m}$ covering $M$ and let $V$ be a neighborhood of $x$ contained in each of the corresponding $V_{m}$. It follows that $f(x, M+N) \cap V=\varnothing$. This is a contradiction.

LEMMA 6. If $H$ is a group and if $t \in T \sim H$ ( $t$ belongs to $T$ but not to $H$ ), then $x \notin \bar{f}(x, t+H)$.

Proof. Suppose $x \in \bar{f}(x, t+H)$. By Lemma 2, there exists a compact set $E$ in $H$ such that $H=E+G$. By Lemma 5 , there exists an element $s$ in $E$ for which $x \in \bar{f}(x, t+s+G)$. Hence, $t+s \in H$ and $t \in H$. This is a contradiction.

Lemma 7. If $H$ is a group and if $t \in T \sim H$, then there exist neighborhoods $V$ of $x$ and $W$ of $t$ such that $f(x, W+H) \cap V=\varnothing$.

Proof. By Lemma 6, there exists a neighborhood $U$ of $x$ for which $f(x, t+H) \cap U=\varnothing$. Choose neighborhoods $V$ of $x$ and $W_{0}$ of 0 so that $W_{0}=-W_{0}$ and $f\left(V, W_{0}\right) \subset U$. Take $W=W_{0}+t$. The proof is completed.

LEMMA 8. If $H$ is a group and if $x$ is recursive with respect to $T$, then $x$ is recursive with respect to $H$.

Proof. Let $U$ be a neighborhood of $x$. Choose neighborhoods $V$ of $x$ and $W$ of 0 so that $W=-W, \bar{W}$ is compact and $f(V, W) \subset U$. Since we may suppose $H \neq T$, we may also suppose that $W+H \neq T$. 
There exists a compact set $F$ in $T$ such that $T=F+H$. Define $E=F \cap[T \sim(W+H)]$. Now $T \sim(W+H)=E+H$. By Lemma 7 , to each element $e$ in $E$ there correspond neighborhoods $V_{\theta}$ of $x$ and $W_{\theta}$ of $e$ so that $f\left(x, W_{\theta}+H\right) \cap V_{\theta}=\varnothing$. Hence, there exists a neighborhood $V^{*}$ of $x$ such that $f(x, E+H) \cap V^{*}=\varnothing$. We may suppose $V \subset V^{*}$, whence $f(x, E+H) \cap V=\varnothing$. There exists an admissible set $A$ in $T$ such that $f(x, A) \subset V$. Hence, $A \cap(E+H)=\varnothing$ and $A \subset W+H$. Define $B=H \cap(A+W)$. Now $f(x, A+W) \subset U$. Since $B$ is an admissible set contained in $H$ and $f(x, B) \subset U$, the proof is completed.

THEOREM 1. If $H$ is a group and if $x$ is recursive with respect to $T$, then $x$ is recursive with respect to $G$.

PROof. The conclusion follows immediately from Lemmas 4 and 8.

Applications. Now let $T$ denote the additive group of either the integers or the reals with its natural topology. Then $G$ is either $T$ or the group of all multiples of a nonzero number. It is clear from Lemma 1 that in this case $H$ is a group.

We consider the following six interpretations of admissibility. A set $A$ in $T$ is said to be admissible provided that:

(1) $A$ contains a sequence marching to $+\infty$;

(2) $A$ contains a sequence marching to $+\infty$ and a sequence marching to $-\infty$;

(3) There exists a positive number $p$ such that each interval of non-negative numbers of length $p$ contains a point of $A$;

(4) There exists a positive number $p$ such that each interval of length $p$ contains a point of $A$;

(5) For each positive number $\epsilon, \lim \sup _{n \rightarrow+\infty} n^{-1} \mu\left\{U_{\epsilon}(A) \cap(0, n)\right\}$ $>0$, where $U_{\epsilon}(A)$ is the $\epsilon$-neighborhood of $A$ in $T, n$ is an integer, $(0, n)$ is the open interval with end points 0 and $n$, and $\mu$ denotes the usual measure of a set in $T$;

(6) For each positive number $\epsilon, \lim \sup _{n \rightarrow+\infty} n^{-1} \mu\left\{U_{\epsilon}(A) \cap(0, n)\right\}$ $>0$ and $\lim \sup _{n \rightarrow+\infty} n^{-1} \mu\left\{U_{\epsilon}(A) \cap(-n, 0)\right\}>0$.

With admissibility defined as $1,2,3,4,5$ or 6 , then the term "recursive" is replaced by "positively recurrent," "recurrent," "positively almost periodic," "almost periodic," "positively strongly recurrent" or "strongly recurrent," respectively.

Theorem 1 now yields immediately the following statement.

Theorem 2. If $x$ is positively recurrent, recurrent, positively almost periodic, almost periodic, positively strongly recurrent or strongly recurrent with respect to $T$, then $x$ has the same property with respect to $G$. 


\section{BIBLIOGRAPHY}

1. P. Erdös and A. H. Stone, Some remarks on almost periodic transformations, Bull. Amer. Math. Soc. vol. 51 (1945) pp. 126-130.

2. W. H. Gottschalk, Powers of homeomorphisms with almost periodic properties, Bull. Amer. Math. Soc. vol. 50 (1944) pp. 222-227.

University of Pennsylvania and UNIVERSITY OF VIRGINIA

\section{A REMARK ON DENSITY CHARACTERS}

\section{EDWIN HEWITT ${ }^{1}$}

Let $X$ be an arbitrary topological space satisfying the $T_{0}$-separation axiom [1, Chap. $1, \S 4$, p. 58]. ${ }^{2}$ We recall the following definition [3, p. 329].

Definition 1. The least cardinal number of a dense subset of the space $X$ is said to be the density character of $X$. It is denoted by the symbol $\Xi(X)$.

We denote the cardinal number of a set $A$ by $|A|$.

Pospísil has pointed out [4] that if $X$ is a Hausdorff space, then

$$
|X| \leqq 2^{2 \Xi(X)} \text {. }
$$

This inequality is easily established. Let $D$ be a dense subset of the Hausdorff space $X$ such that $|D|=\Xi(X)$. For an arbitrary point $p \in X$ and an arbitrary complete neighborhood system $v_{p}$ at $p$, let $\mathcal{D}_{p}$ be the family of all sets $U \cap D$, where $U \in V_{p}$. Thus to every point of $X$, a certain family of subsets of $D$ is assigned. Since $X$ is a Hausdorff space, $\mathcal{D}_{p} \neq \mathcal{D}_{q}$ whenever $p \neq q$, and the correspondence assigning each point $p$ to the family $\mathcal{D}_{p}$ is one-to-one. Since $X$ is in one-to-one correspondence with a sub-hierarchy of the hierarchy of all families of subsets of $D$, the inequality (1) follows.

It may be remarked in passing that the inequality (1) does not obtain for all $T_{1}$-spaces. Let $\mathfrak{m}$ be a cardinal number greater than $2^{c}$, where $\mathfrak{c}=2 \aleph_{0}$. Let $Z$ be a $T_{1}$-space of cardinal number $\mathfrak{m}$ and with the property that the only closed proper subsets of $Z$ are finite or

Received by the editors March 5, 1946.

${ }^{1}$ Post-service fellow of the John Simon Guggenheim Memorial Foundation.

2 Numbers in brackets refer to the Bibliography at the end of the paper. 